\section{Dodging defences}

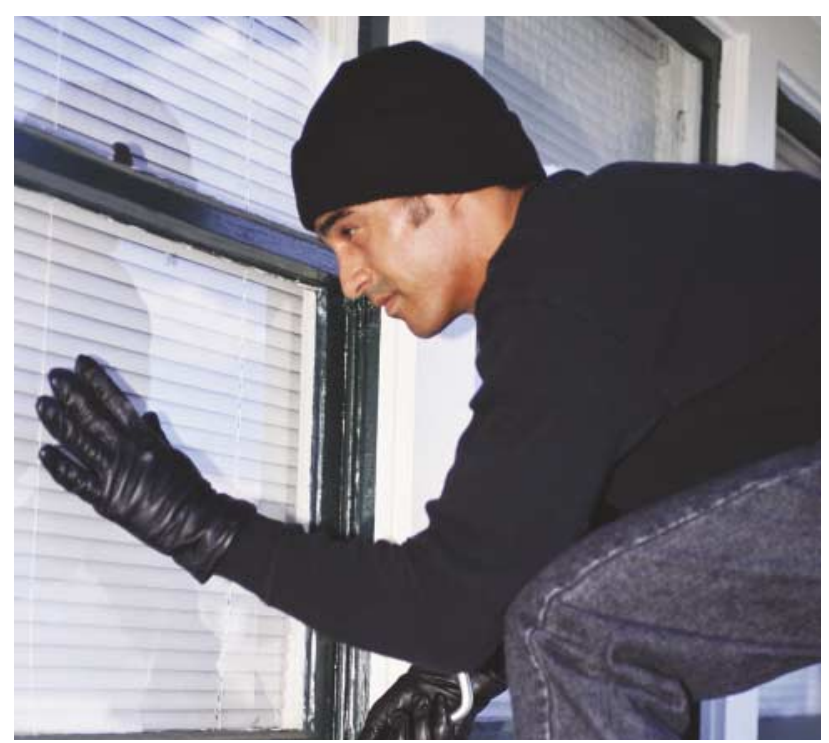

In 1997, an outbreak of deadly influenza virus hit the live poultry markets of Hong Kong. Having jumped straight from chickens to humans, this virus strain, known as H5N1/97, killed six of the 18 people known to be infected. A report in Nature Medicine now reveals the secret weapon of the virus.

Postmortems showed that the victims had massive levels of virus in their lungs and that they had probably died as a result of the overproduction of cytokines, such as interferons (IFNs) and tumour-necrosis factor (TNF). These cytokines have potent antiviral effects, which led Heui Seo and colleagues to wonder if the lethal virus strain might somehow be resistant to antiviral cytokines. Remarkably, they found that the replication of $\mathrm{H} 5 \mathrm{~N} 1 / 97$ in pig cells was completely unaffected by the addition of IFN- $\alpha$, IFN- $\gamma$ or TNF, at any dose, whereas the replication of common influenza subtypes was inhibited.
So, how does $\mathrm{H} 5 \mathrm{~N} 1 / 97$ dodge the antiviral effects of cytokines? Several previous studies have reported that influenza-virus non-structural protein 1 (NS1) dampens the host response to IFN- $\alpha / \beta$, which makes it a promising candidate resistance gene. The authors inserted the NS1 gene of H5N1/97 into another non-lethal influenza virus, H1N1. They found that replication of the wild-type H1N1 virus was inhibited completely by antiviral cytokines, whereas replication of the recombinant virus was unabated, which confirmed the essential role of NS1 in immune evasion.

A comparison of the amino-acid sequences of NS1 from various influenza viruses showed that the $\mathrm{H} 5 \mathrm{~N} 1 / 97 \mathrm{NS} 1$ is unique in that it has a glutamic acid at position 92. All of the other known influenza-virus strains have an aspartic acid at this position. To test the importance of this residue, the Glu92 of H5N1/97 NS1 was changed to an aspartic acid

\title{
Seeking the selectors
}

The nature of the peptides that mediate thymic positive selection has been much debated. Reporting in the September issue of Immunity, Santori and colleagues have characterized peptides that induce positive selection using bioassay- and bioinformatics-based approaches. Reassuringly, both lines of investigation picked out the same two peptide ligands.

Positive selection is the process by which $\mathrm{CD}^{+} \mathrm{CD}^{+}$double-positive thymocytes that interact with self-peptide-MHC complexes are selected to survive and mature. This developmental checkpoint is thought to ensure that all mature T cells can interact with self-MHC molecules. But, what makes a peptide a positive selector and how common are they?

The authors addressed these issues by characterizing naturally occurring peptides that mediate positive selection in a T-cell receptor (TCR)-transgenic model. OT-1 mice express a TCR that is specific for an ovalbumin (OVA) peptide presented by the MHC class I molecule $\mathrm{H} 2-\mathrm{K}^{\mathrm{b}}$.

In the first approach, bound peptides were extracted from $\mathrm{H} 2-\mathrm{K}^{\mathrm{b}}$ molecules that had been purified from various cell lines and tissues. Peptide fractions were then tested for activity in an assay for positive selection - the 'co-receptor-dulling assay'. This measures the ability of a peptide ligand to induce the downregulation of expression of the CD4 or CD8 co-receptors on thymocytes, which is an in vitro correlate of positive selection. Candidate peptides in positive fractions were sequenced, then synthesized and tested for their ability to induce positive selection in fetal thymic organ culture. This process identified two positively selecting peptides: one from $\beta$-catenin $\left(\mathrm{Catnb}_{329-336}\right)$ and the other from $\mathrm{F}$-actin capping protein $\left(\right.$ Cappa $\left._{\text {92-99 }}\right)$.

The Catnb ${ }_{329-336}$ and Cappa $1_{92-99}$ peptides have some structural homology to the OVA peptide that is the OT-1 TCR agonist. So, can positively selecting ligands be predicted on the basis of sequence homology? The second bioinformaticsbased approach tested this theory. A database of predicted mouse MHC ligands was assembled, then algorithms were devised to select ligands that were similar to the OVA peptide at key TCR contact sites. Only ligand peptides for which the parent protein is expressed in the thymus were considered. Seven candidate peptides were identified which were then tested for their ability to bind to $\mathrm{H} 2-\mathrm{K}^{\mathrm{b}}$ and for activity in the co-receptor-dulling assay. Ruling out peptides that are not actually presented by $\mathrm{H} 2-\mathrm{K}^{\mathrm{b}}$ in the thymus gave an end result of two peptides - Catnb $_{329-336}$ and Cappa $1_{92-99}$ - which, remarkably, are the same peptides that were identified by the bioassay-based approach.

So, it seems that the peptides that induce positive selection are rare and have key TCR contact residues in common. Moreover, this study sets an important precedent for the general use of bioinformatics to predict the ligands that mediate positive selection.

Jennifer Bell

(D) References and links

ORIGINAL RESEARCH PAPER Santori, F. R

et al. Rare, structurally homologous self-peptides promote thymocyte positive selection. Immunity 17, 131-142 (2002) FURTHER READING Hogquist, $K$. A. Signal strength in thymic selection and lineage commitment. Curr. Opin. Imymunol. 13, 225-231 (2001) WEB SITES

Stephen Jameson's lab:

http://www.micab.med.umn.edu/faculty/Jameson.html Kristin Hogquist's lab:

http://www.micab.med.umn.edu/faculty/Hogquist.html Stanislav Vukmanovic's lab: http://www.med.nyu.edu/research/vukmas01.htm 
by site-directed mutagenesis. The mutated NS1 was then inserted into the H1N1 virus. This recombinant virus was susceptible to the effects of IFN- $\alpha$, IFN- $\gamma$ and TNF, which indicates that Glu92 of NS1 is essential for the resistance of $\mathrm{H} 5 \mathrm{~N} 1 / 97$ to antiviral cytokines. The importance of NS1 for the pathogenicity of $\mathrm{H} 5 \mathrm{~N} 1 / 97$ was confirmed by experiments in pigs.

This study has pinpointed a lethal single-amino-acid change that renders a virus completely resistant to antiviral cytokines. But, the mechanism by which Glu92 of NS1 confers this resistance is not clear. With new strains of cytokine-resistant influenza virus lurking in Hong Kong's poultry markets, this is an important question for future research.

Jennifer Bell

(2) References and links original ReSEARch PAPER Heui Seo, $S$. Hoffmann, E. \& Webster, R. G. Lethal H5N1 influenza viruses escape host anti-viral cytokine responses. Nature Med. 8, 950-954 (2002)

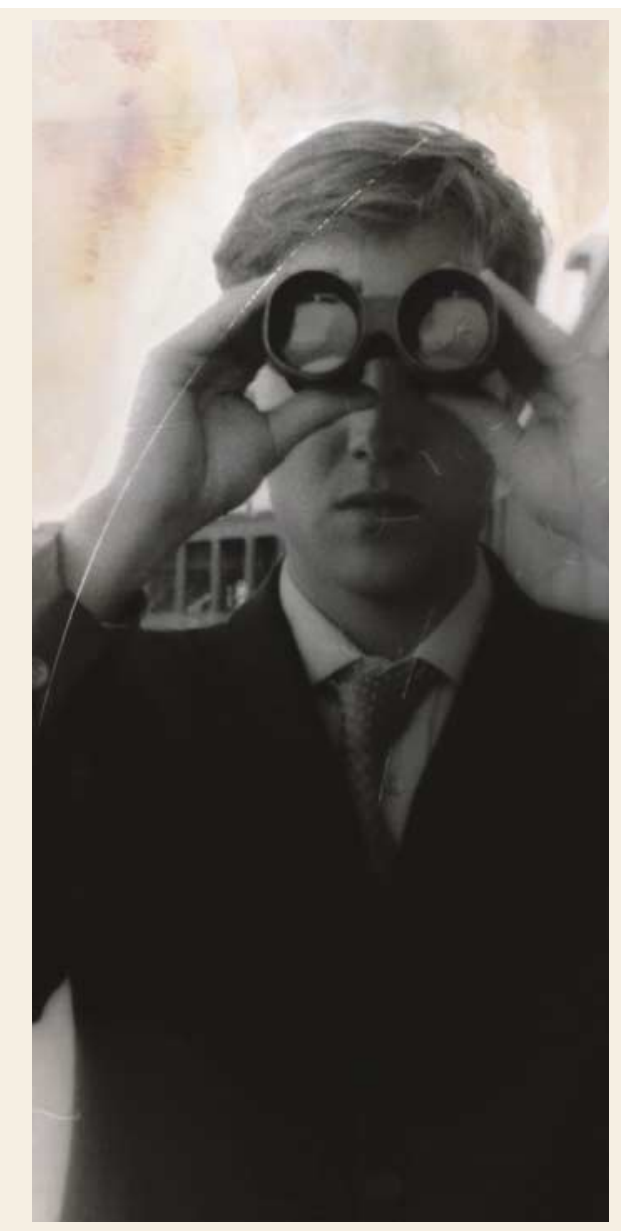

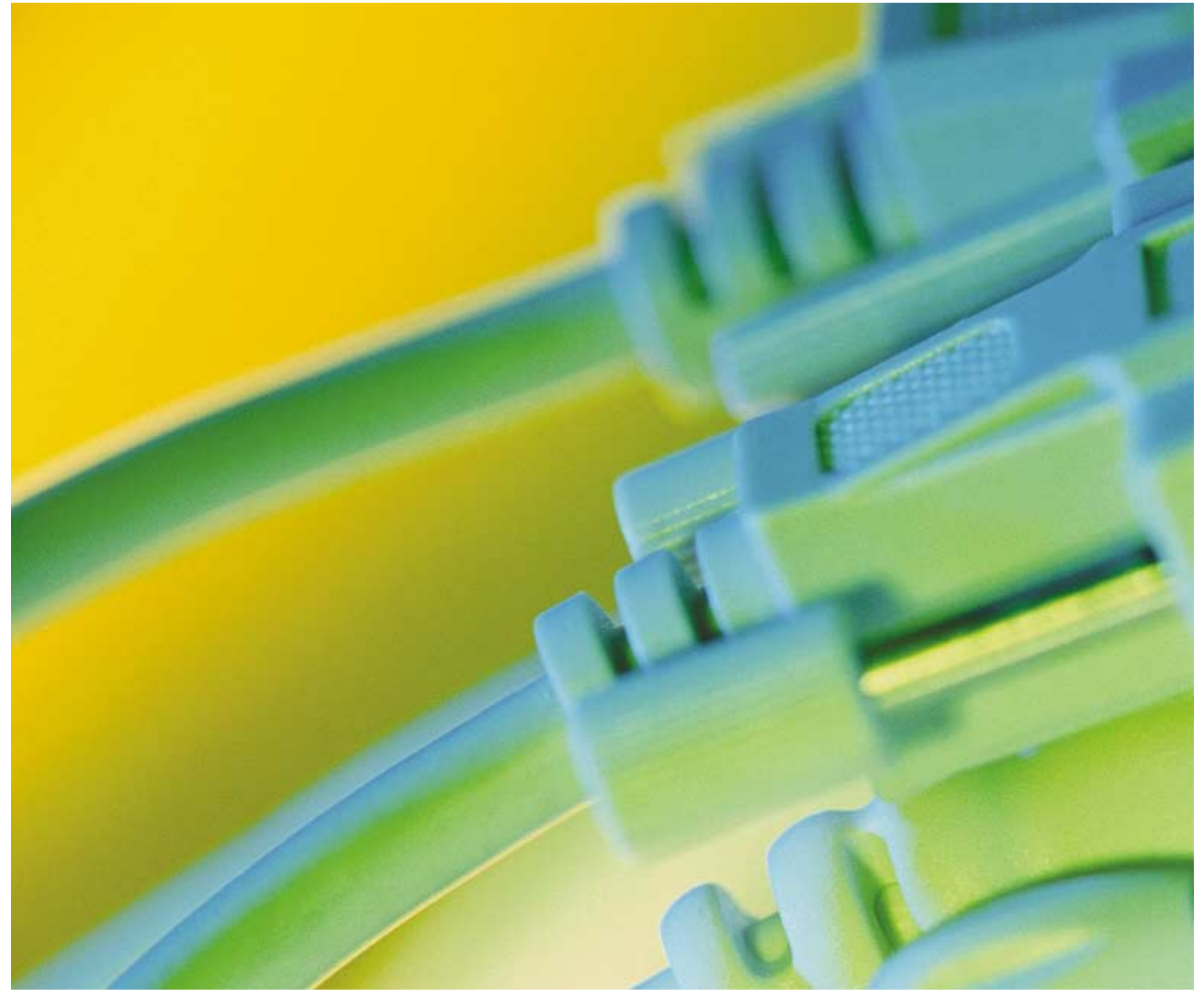

AUTOIMMUNITY

\section{Mast cells make the connection}

$\mathrm{K} / \mathrm{BxN}$ mice are a T-cell-receptor-transgenic model of rheumatoid arthritis in which autoantibodies specific for a ubiquitously expressed self-antigen (MHC class II) are produced. The disease can be transferred by autoantibody-containing serum. Complement, Fc receptors and cytokines - such as tumour-necrosis factor (TNF) and interleukin-1 (IL-1) — are known to be involved in disease pathogenesis in the mouse model. But, what is the cellular connection between these soluble factors and the pathogenic neutrophil infiltration of the joints that is observed in the mouse model, as well as in humans? Lee et al. think they have the answer. In a recent Science paper, they propose that mast cells are the missing link.

Mast cells are a good candidate because they are resident in the synovium and have the functional capability to produce TNF and IL-1, as well as other inflammatory mediators, matrix proteases and neutrophil chemoattractants. To analyse the potential role of mast cells, the authors used two strains of mice that lack tissue mast cells $-\mathrm{Sl} / \mathrm{Sl}^{\mathrm{d}}$ and $\mathrm{W} / \mathrm{W}^{\mathrm{v}}$ mice - owing to a deficiency of the transmembrane form of stem-cell factor (SCF) or mutations in the SCF receptor c-Kit, respectively. Both mouse strains had markedly reduced clinical and histological signs of arthritis compared with wild-type littermates after transfer of $\mathrm{K} / \mathrm{BxN}$ serum.

However, it could be that SCF-c-Kit signalling has some role in disease pathogenesis through another cellular system. To establish that the resistance to arthritis was due to the mast-cell deficiency, complementation analysis was used to assess the specific role of mast cells. Mast-cell engraftment was shown to restore arthritis susceptibility to $\mathrm{Sl} / \mathrm{Sl}^{\mathrm{d}}$ and $\mathrm{W} / \mathrm{W}^{\mathrm{v}}$ mice.

So, if mast cells are the cellular missing link, then mast-cell degranulation must be the functional link. After intraperitoneal transfer of $\mathrm{K} / \mathrm{BxN}$ serum to $\mathrm{C} 57 \mathrm{BL} / 6 \mathrm{~J}$ mice, mast-cell degranulation in the affected joints was shown to occur before any clinical evidence of inflammation, whereas no degranulation could be seen at other anatomical locations. This indicates that mast cells are involved in the initiation of inflammation. Also, degranulation continues during chronic arthritis, which indicates that mast cells might be involved in disease maintenance as well.

As for any animal model, arthritis in $\mathrm{K} / \mathrm{BxN}$ mice is not an exact replica of human rheumatoid arthritis - for example, in terms of the joints that are affected and the spectrum of antibodies that is produced, as well as the fact that the disease is more aggressive in mice. However, the similarities are sufficient to indicate that mast cells probably have a crucial role in human arthritis also. This is supported by the fact that synovial sections from humans contain a large number of mast cells, as well as immune complexes, complement fragments and SCF.

Kirsty Minton

(2) References and links

ORIGINAL RESEARCH PAPER Lee, D. M. et al. Mast cells: a cellular link between autoantibodies and inflammatory arthritis. Science 297 1689-1692 (2002)

FURTHER READING Kouskoff, V. et al. Organ-specific disease provoked by systemic autoimmunity. Cell $\mathbf{8 7}, 811-822$ (1996) 